\title{
Erratum to: Experience of 11 years use of cidofovir in recurrent respiratory papillomatosis
}

\author{
M. Graupp • M. Gugatschka $\cdot$ K. Kiesler • \\ E. Reckenzaun · G. Hammer · G. Friedrich
}

Published online: 31 May 2013

(C) Springer-Verlag Berlin Heidelberg 2013

Erratum to: Eur Arch Otorhinolaryngol (2013)

270:641-646

DOI 10.1007/s00405-012-2221-2

There is a discrepancy in the figures and the related captions in the online published article. The corrected figures and captions are presented below.
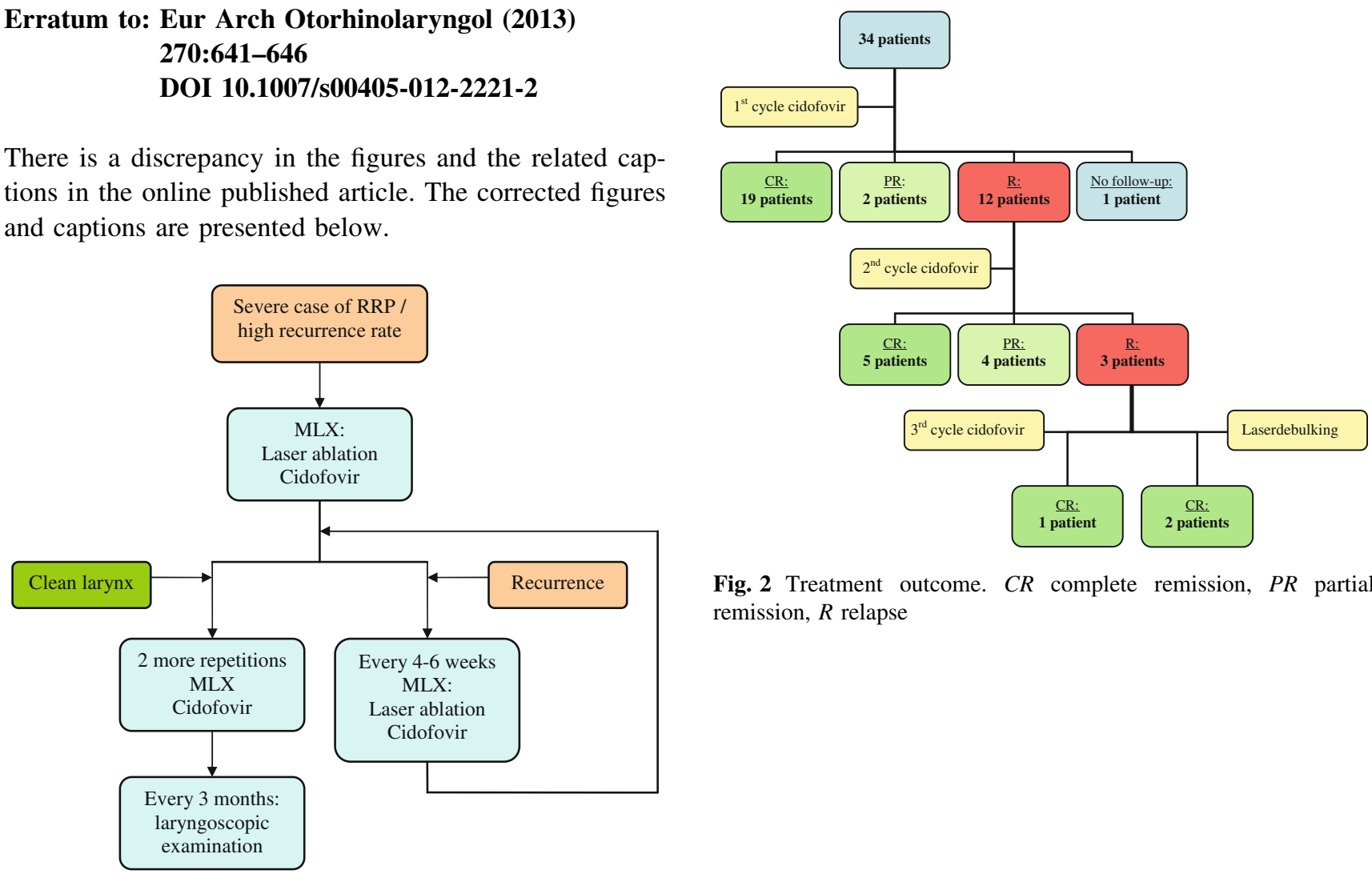

Fig. 2 Treatment outcome. $C R$ complete remission, $P R$ partial remission, $R$ relapse

Fig. 1 Standard protocol

The online version of the original article can be found under doi:10.1007/s00405-012-2221-2.

M. Graupp $(\bowtie) \cdot$ M. Gugatschka $\cdot$ K. Kiesler .

E. Reckenzaun · G. Hammer · G. Friedrich Department of Phoniatrics, ENT University Clinic, Speech and Swallowing, Medical University Graz, Auenbruggerplatz 26-28, 8036 Graz, Austria e-mail: matthias.graupp@gmx.at 